\title{
De responsabilidad social a sostenibilidad corporativa: una revisión actualizada
} From Corporate Social Responsibility to Corporate Sustainability: an updated Review

Jaime González Masip

Universidad Complutense de Madrid

jgmasip@ucm.es

Pedro Cuesta Valiño

Universidad de Alcalá

pedro.cuesta@uah.es
González Masip, J. y Cuesta Valiño, P. (2018)

De responsabilidad social a sostenibilidad corporativa: una revisión actualizada

Revista Internacional de Investigación en Comunicación aDResearch ESIC. No 17 Vol 17

Primer semestre, enero-junio 2018 · Págs. 46 a 71

DOI: 17.7263/ADRESIC.017.003 
RESUMEN

\section{Clasificación JEL: M110, M140, M330 Palabras clave:} Responsabilidad social corporativa, RSC, sostenibilidad corporativa, ciudadanía corporativa, Rendimiento social, respuesta social, responsabilidad medioambiental, RMC.
La responsabilidad social corporativa (RSC) es una disciplina de alto interés actualmente para académicos y profesionales, pero que, sin embargo, no goza todavía de consenso en cuanto a definición y enfoques fundamentales. A lo largo de las últimas décadas, han aparecido nuevos componentes de la RSC, así como otros conceptos derivados que incorporan, en la mayoría de los casos y al hilo de la creciente concienciación por el deterioro natural, una dimensión medioambiental muy marcada que condiciona el resto de sus componentes. A pesar de tener su origen en las expectativas y demandas de individuos y grupos sociales, la responsabilidad corporativa es fuente de ventajas competitivas, entre las que se incluyen una mejora en la imagen y la reputación de las empresas que la implementan y ejecutan positivamente, por lo que resultan de alto interés para las mismas. El objetivo de este trabajo, basado en el contenido de la tesis doctoral de González-Masip (2017), es la realización de una revisión teórica, fundamentada principalmente en artículos de investigación, del concepto de responsabilidad social corporativa, identificando algunos de sus fundamentos, componentes y evolución temporal, analizando su relación con la responsabilidad medioambiental corporativa e incluyendo una revisión otros conceptos relacionados con RSC, como sostenibilidad corporativa o ciudadanía corporativa. Este articulo ayuda a comprender el fenómeno de la responsabilidad empresarial, la actuación social corporativa y sus diferentes derivados, identificando las relaciones y conexiones existentes entre similares conceptos para conseguir una visión general de la cuestión. También permite comprender los diversos planteamientos de imagen social corporativa que la empresa puede reflejar a través de sus iniciativas de comunicación y que influyen en el nivel de aceptación y efectividad de las mismas entre consumidores y otros grupos de interés.
ABSTRACT

\section{JEL Classification: M110, M140, M330 Key words:}

Corporate social responsibility, CSR, corporate sustainability, corporate citizenship, corporate social performance, corporate social responsiveness, corporate environmental responsibility, CER.
Corporate Social Responsibility (CSR) is a discipline of high interest to academics and practitioners. Nevertheless, there is no consensus yet on a definition and fundamental approaches. Over the last decades, new components of CSR have appeared, together with other derivative concepts that incorporate a very marked environmental dimension that conditions the rest of the ideas, as a result of the increasing awareness of the environmental deterioration. Despite its origin in the expectations and demands of individuals and social groups, corporate responsibility is the source of competitive advantages, which include the image and reputation improvement for the companies, so the positive implementation of the CSR turns out to be of high interest for enterprises. The aim of this paper, based on the content of the doctoral thesis by González-Masip (2017), is to carry out a theoretical review of the concepts of corporate social responsibility, identifying some of its foundations, components and temporal evolution, analyzing its relationship with corporate environmental responsibility and a review of other concepts related to CSR, such as corporate sustainability or corporate citizenship. Based fundamentally on research papers, this article helps to understand the phenomenon of corporate responsibility, corporate social action and its various derivatives, identifying the relationships and connections between the various concepts to get an overview of the issue. It also allows us to understand the diverse proposals of corporate social image that the company can reflect through its communication initiatives, and that influence the level of acceptance and effectiveness among consumers and other stakeholders 


\section{Introducción}

La responsabilidad social corporativa (RSC) es una disciplina de estudio que ha captado la atención de académicos y profesionales de manera creciente en las últimas décadas (Aguinis y Glavas, 2012; Waddock et al., 2002). Está relacionada con aquellas actuaciones de empresas que puedan contribuir al bien social, sobrepasando los propios intereses de la empresa y sus obligaciones legales o regulatorias (McWilliams y Siegel, 2001). Tanto responsabilidad social corporativa como responsabilidad medioambiental corporativa están relacionadas con el compromiso de las empresas en sus ámbitos sociales y entornos naturales de operación (Bansal y Roth, 2000). Retratan la intención de la empresa por generar un beneficio que va más allá del económico, atendiendo a las necesidades y exigencias de los grupos de interés, que son aquellos individuos y colectivos afectados por la actividad de la empresa o con influencia en la misma (Freeman, 1984) que actúan en representación de la sociedad y el medio natural.

Las expectativas de las personas de un comportamiento socialmente responsable por parte de las empresas han aumentado significativamente desde hace varias décadas. Los individuos y colectivos esperan de manera creciente que las corporaciones sean social y medioambientalmente responsables frente a fenómenos como la degradación forestal, la sobre-explotación o destrucción de recursos naturales o el cambio climático en el plano medioambiental, y derechos laborales o derechos humanos en el plano social (Auld et al., 2008). En general, esta nueva ola requiere que las empresas integren actuaciones de naturaleza filantrópica en su misión como organización y las considere capacidades clave (Fox, 2007). La RSC evoluciona hacia una realidad práctica, y las empresas se replantean su pa- pel en la sociedad y redefinen su perfil ético de actuación (Lichtenstein et al., 2004).

Numerosos estudios demuestran que la responsabilidad social es fuente de ventaja competitiva y oportunidades para la empresa (Baron, 2001; Gallardo-Vázquez y Sánchez-Hernández, 2014; Porter y Kramer, 2006; Weber, 2008), y por lo tanto, afecta positivamente a los resultados económicos (Albinger y Freeman, 2000; Waddock y Graves, 1997). «En el largo plazo, las empresas más exitosas son aquellas que pueden alcanzar tanto una respuesta social, como un buen rendimiento económico» (Ackerman, 1973, p. 88). De acuerdo a Weber (2008), los beneficios obtenidos por las empresas por la adopción de RSC en su estrategia de negocio provienen de, entre otros: los efectos positivos generados sobre la imagen de la empresa y su reputación (Fombrun y Shanley, 1990); el aumento del beneficio económico, mediante la diferenciación, por el acceso a nuevos mercados con clientes sensibles a las iniciativas responsables (Bagnoli y Watts, 2003; Baron, 2008) o a mercados más rentables; y la reducción de los riesgos generados por escándalos, presiones o boicots.

Cuando las empresas adoptan iniciativas de RSC consiguen mejoras en comportamiento, apoyo y vinculación de los grupos de interés con la empresa (Du et al., 2010). Las comunicaciones o anuncios que incluyen mensajes con causas sociales provocan actitudes más favorables del consumidor hacia la compañía que aquellos que no los incluyen (Nan y Heo, 2007). Este efecto positivo se aprecia en la evaluación de la marca, evaluación de productos y de nuevos productos, e influyen favorablemente en el impacto de las crisis o escándalos, especialmente cuando los consumidores están familiarizados y son sensibles a los aspectos de RSC (Klein y Dawar, 2004). En general, las percepciones sobre las actuaciones en 
RSC provocan efectos positivos en los niveles de satisfacción de los clientes, de reputación y de valor de marca (Hsu, 2012).

La RSC influye en la intención de compra de los consumidores, afectando en ello factores específicos de la empresa, como puede ser el caso de la industria a la que pertenece (McWilliams et al., 2006), aspectos de RSC en que la empresa se centra, y el perfil del consumidor (como grupo de interés afectado) y sus creencias y percepciones (Sen y Bhattacharya, 2001). Las motivaciones de la actividad de RSC de la empresa deben ser transparentes, sinceras y no ambiguas, y sin generar sospechas sobre su veracidad, o de lo contrario la imagen de la compañía se ve afectada negativamente (Yoon et al., 2006). Aunque la comunicación de las iniciativas éticas y socialmente responsables de las empresas generan beneficios, también generan una actitud crítica por parte del público objetivo, especialmente en ciertos tipos de industrias como las de armamento, alcohol o tabaco (Morsing y Schultz, 2006). Los consumidores pueden percibir cierto nivel de hipocresía cuando la comunicación de la RSC no coincide con la realidad de la empresa, dañando su actitud hacia la misma (Wagner et al., 2009), más aun considerando que la rendición de cuentas sobre la actividad en RSC es objeto de un alto grado de escepticismo al considerarse un modo más comunicación publicitaria (Siegel y Vitaliano, 2007). Las empresas deben trasladar de manera eficaz su posicionamiento social a sus grupos de interés y contrarrestar los posibles prejuicios existentes en relación a la RSC (Du et al., 2010). Acertar con el enfoque adecuado es necesario para no correr el riesgo de impactar negativamente en la intención de compra (Sen y Bhattacharya, 2001).

A pesar de la relativa antigüedad del concepto de RSC, todavía no existe un consenso sobre su definición y enfoque adecuado (Aguinis y Glavas, 2012; Sheehy, 2015). Numerosos autores interpretan la RSC analizando posibles perspectivas, dimensiones, niveles o conceptos derivados (Carroll, 1979; Garriga y Melé, 2004; Wood, 2010). La evolución del entorno y sus exigencias ha generado nuevas interpretaciones de la responsabilidad corporativa que acuñan términos como sostenibilidad corporativa o triple línea base, con una mayor presencia del componente de responsabilidad medioambiental y la consideración de la actuación en el largo plazo (Dahlsrud, 2008; Park y Levy, 2014; Van Marrewijk, 2003; Waddock y Bodwell, 2004).

El objetivo de este artículo es la realización de una revisión teórica del concepto de responsabilidad social corporativa, identificando algunos de sus fundamentos, componentes, evolución temporal y relaciones existentes, hasta derivar en conceptos más contemporáneos, relacionados con el anterior, como sostenibilidad corporativa o ciudadanía corporativa. Estos conceptos resultan de utilidad para comprender el fenómeno de la responsabilidad empresarial, la actuación social y sus diferentes derivados. Esta revisión conceptual pretende contribuir al entendimiento de diferentes perspectivas y planteamientos que se pueden encontrar en el estudio del fenómeno de la actividad social de las empresas para poder plantear con mayor precisión las propuestas de investigación en el ámbito del marketing y la comunicación, e identificar posibles desviaciones en el ajuste entre el posicionamiento social percibido de las empresas y su estrategia de comunicación.

Este trabajo se basa en los contenidos de la tesis doctoral de González-Masip (2017) relacionada con el efecto de prácticas de responsabilidad social y medioambiental corporativa en el talento presente en la empresa. El contenido del 
artículo se estructura comenzando con un estudio del concepto de la RSC y sus perspectivas de análisis; a continuación se estudian otros conceptos derivados como la respuesta social, el rendimiento social y la responsabilidad medioambiental corporativa; y por último se presentan y comparan conceptos de más reciente creación, en muchos casos fundamentados en el componente medioambiental de la responsabilidad corporativa.

\section{La responsabilidad social corporativa}

Existe un permanente debate sobre una posible definición de responsabilidad social corporativa (RSC) sin que académicos, profesionales o directivos reconozcan una definición única, clara y sencilla de la misma, generando en ocasiones cierto grado de confusión (Aguilera et al., 2007; Aguinis y Glavas, 2012; Bauman y Skitka, 2012; Dahlsrud, 2008; Garriga y Melé, 2004; McWilliams et al., 2006; Sheehy, 2015; Van Marrewijk, 2003). En general se ha producido una evolución en la interpretación de la RSC a lo largo de décadas. RSC significa diferentes cosas para diferentes personas (Lyon y Maxwell, 2008) debido a varios motivos.

En primer lugar, la RSC se ha desarrollado al amparo de diversas teorías (Lindgreen y Swaen, 2010) entre las que se encuentran la Teoría de la Agencia, la Teoría Institucional, la Teoría de Recursos y Capacidades (Resource-Based View), la Teoría de Grupos de Interés (o stakeholders), la Teoría de la Administración (Lindgreen y Swaen, 2010), la Teoría de Señales (Behrend et al., 2009; Greening y Turban, 2000; D. A. Jones et al., 2014), o la Teoría de la Identidad Social (Brammer et al., 2007; Greening y Turban, 2000), entre otras. La elección de las teorías para cada estudio depende del criterio de cada investigador y el enfoque según el cual se pretende analizar el fenómeno o concepto de la responsabilidad corporativa.

Por otra parte, muchas definiciones presentan cierta parcialidad, en ocasiones para justificar de manera eficiente determinadas actuaciones empresariales o investigaciones (Dahlsrud, 2008). Los planteamientos de la RSC encajan en los objetivos de diversas disciplinas involucradas en la gestión empresarial, como gestión de la calidad, marketing, comunicación, finanzas, ingeniería o gestión de recursos humanos, entre otras. Algunas aproximaciones al concepto de RSC se han orientado según los intereses y objetivos de la disciplina en concreto (Van Marrewi$j k$, 2003). Las investigaciones orientadas a aspectos prácticos tienden a aportar definiciones que muestran guías de aplicación y utilidad, mientras que las investigaciones de tipo más académico se caracterizan por enfoques más holísticos, complejos y filosóficos (Montiel y DelgadoCeballos, 2014).

Otro factor que motiva la falta de consenso sobre una única definición se debe a las múltiples facetas (Brammer et al., 2007) y las diferentes perspectivas y dimensiones desde los que se puede analizar el concepto (McWilliams et al., 2006), dando como consecuencia también una falta de homogeneidad en los métodos de medición en las investigaciones (Aguinis y Glavas, 2012).

\subsection{El concepto de responsabilidad social corporativa}

Una empresa es considerada una entidad creada y facultada por las instituciones públicas y sociales para actuar como un individuo (Kilcullen y Kooistra, 1999). Como referencia de partida, y considerando esta similitud con las personas, la RSC pretende responder a la pregunta «ide qué 
es responsable la empresa?» (Maignan et al., 1999, p. 457). Se puede definir responsabilidad social corporativa como «acciones [que realiza una empresa] que parecen promover el bien social, más allá de intereses de la empresa y de lo requerido por la ley» (McWilliams y Siegel, 2001, p. 117), o como «políticas claramente articuladas y comunicadas, y prácticas de corporaciones que reflejan la responsabilidad de las empresas hacia el bien social en su contexto más amplio» (Matten y Moon, 2008, p. 405). La RSC presenta un enfoque amplio que involucra a las empresas en corrientes de alto nivel, con planteamientos sobre la faceta ética de su participación en la economía y la sociedad (Knudsen, 2013). Incorpora el componente de iniciativa de carácter voluntario y de compromiso, lo que supone una especie de marco auto-regulatorio para las empresas, acompañado de una faceta relacionada con las cada vez más abundantes normativas y prácticas de recomendado cumplimiento (Sheehy, 2015). Analizando sus posibles etiquetas, S. Rahman (2011) observa en las definiciones de RSC la existencia de diez dimensiones que permiten identificar las bases del concepto: obligación hacia la sociedad, involucración de grupos de interés, mejora de la calidad de vida, desarrollo económico, practica de éticas de negocio, cumplimiento con la ley, voluntariado, derechos humanos, protección del medioambiente, así como transparencia y rendición de cuentas.

La RSC está muy relacionada con las expectativas de la sociedad sobre el comportamiento de la empresa respecto a sus responsabilidades sociales (Carroll, 1979). Dicho comportamiento es demandado a través de los grupos de interés y está moralmente justificado, por lo que es legítimamente exigible a una empresa (Whetten et al., 2002). Grupos de interés son «cualquier grupo o individuo que puede afectar o es afectado por la consecución de los objetivos por parte de una empresa» (Freeman, 1984, p. 46). La idea básica es que empresas y sociedad no son entidades separadas, sino que están interrelacionadas, y la sociedad tiene ciertas expectativas sobre comportamientos y resultados (e impactos) de la empresa (Wood, 1991). La RSC se refiere a esta relación de empresa y sociedad, que implica cuestiones sobre derechos, justicia, y cómo la empresa afecta al bienestar humano (Bauman y Skitka, 2012). Por otra parte, su componente de voluntariedad atribuye a la RSC matices de altruismo en el comportamiento de la empresa, es decir, la asunción por parte de ésta de iniciativas de redistribución de la riqueza sin la existencia de presión previa por parte de grupos de interés (Baron, 2001). Altruismo puede definirse como «la intención de beneficiar a otros como una expresión de valores internos, independientemente del refuerzo social o motivacional» (Price et al., 1995, p. 257).

Los grupos de interés, cuyas demandas deben ser estudiadas y atendidas, condicionan la interpretación dada a la RSC. Frente a perspectivas que afirman que el principal propósito de la empresa es crear beneficio económico, y por lo tanto no deben preocuparse por otros asuntos sociales (Friedman, 1970; Levitt, 1958), las interpretaciones contemporáneas del concepto de RSC tienden a considerar la necesidad de las empresas de internalizar los posibles efectos negativos que pueda ocasionar su operativa en el entorno de manera preferente, frente a compensarlos externamente con la realización de contramedidas (Auld et al., 2008).

«La responsabilidad social corporativa se relaciona principalmente con el logro de los resultados de decisiones organizacionales sobre asuntos o problemas especificos que (por algún referente de 
tipo normativo) tienen efectos beneficiosos en lugar de efectos adversos sobre los grupos de interés de la corporación. La corrección de los resultados de la acción corporativa ha sido el enfoque principal de la RSC» (Epstein, 1987, p. 104).

Se puede decir que la RSC consiste en la búsqueda del bien general de la sociedad involucrando de manera intensa en su planteamiento a un amplio conjunto de grupos de interés (Barnett, 2007). Respecto a un enfoque de estrategia empresarial clásico, los planteamientos de la RSC tienen en consideración la generación de valor (Hussain, 1999) y bienestar para la sociedad, y contempla las relaciones con los grupos de interés como medio para alcanzar tal fin.

Para la RSC, la dimensión de medioambiente está considerada una parte importante y específica englobada dentro de un concepto mayor de responsabilidad social (Lynes y Andrachuk, 2008; Montiel, 2008; Willums, 1999, en Orlitzky et al., 2003; Post et al., 2011), y restringida a la manera en que las empresas y su industria se relacionan con el entorno natural (Nicholls y Kang, 2012). Así, medioambiente es una dimensión más, no principal del constructo, junto con otros conceptos como derechos humanos, derechos de los trabajadores o relaciones con la comunidad y los proveedores (Watts y Holme, 2000).

\subsection{Evolución histórica del concepto}

El concepto de responsabilidad social corporativa (RSC) encuentra sus orígenes en trabajos de autores como Dodd (1932) que, haciendo referencia a las empresas y sus directivos, les asigna una responsabilidad hacia la sociedad, más allá de los accionistas, residiendo la prueba de esta existencia en que las propias leyes que amparan el funcionamiento de las empresas lo hacen por el beneficio que aportan a la sociedad en su conjunto (Dodd, 1932, en Cochran, 2007). Por su parte, Bowen (1953) analiza las responsabilidades que afectan a los directivos respecto a la sociedad, mencionando explícitamente el concepto de responsabilidad social. También en los años cincuenta, Boulding (1956) sienta las bases del rendimiento social de las corporaciones mencionando la interacción y el impacto de las empresas, como sistemas abiertos, en su entorno, en el cual influyen de manera constante. Más tarde, Davis (1960) relaciona la responsabilidad de los directivos con su poder, la capacidad de afectación en trabajadores, desempleo y la honestidad de las acciones de la empresa y el carácter voluntario de la adopción de uno u otro tipo de actitudes. Ese mismo año, Frederick (1960) reflexiona sobre la capacidad de las empresas para crear valor social más allá de la riqueza económica, destacando nuevamente la figura del directivo de negocios como pieza clave en la deriva ética de la empresa y el rol social de ésta en la sociedad.

Analizando la evolución histórica de los enfoques de RSC, se observa que en los años cincuenta predomina una interpretación como «obligación a la sociedad»; en los años sesenta abunda el enfoque de «relación entre corporación y sociedad»; y en los años setenta se incorporan aspectos legales y filosóficos, así como los grupos de interés (S. Rahman, 2011). A finales de la década de los años sesenta, McGuire (1969) identifica cuatro aproximaciones al concepto de RSC que aportan información para una comprensión del mismo: tradicional (enfoque neoclásico que dice que la RSC no tiene lugar en los negocios), liberal (la RSC sirve a la propia empresa), responsable (RSC es lo correcto a hacer, aunque puede que no compense), y una última aproximación denominada confusa (la ética justifica la RSC, y a través de esta la empresa obtiene compensación de manera extraña). Durante los 
años ochenta y noventa, se frena, aunque no detiene, el interés por definir el concepto de RSC y se comienzan a estudiar y proponer conceptos alternativos o componentes del mismo, como la respuesta social o el rendimiento social corporativo (Carroll, 1979).

Es en la primera década del siglo XXI, especialmente a partir del trabajo de Carroll (1999), cuando se consolida el componente medioambiental como parte integrante del concepto de responsabilidad social corporativa (Dahlsrud, 2008). La vinculación de la dimensión medioambiental respecto a la responsabilidad corporativa puede generar confusión. En ocasiones la responsabilidad medioambiental goza de identidad propia e independiente, asignando en ese caso a la responsabilidad social un alcance específico hacia las personas (sin considerar aspectos medioambientales). En otras ocasiones se considera medioambiente como un componente más de la responsabilidad de la empresa hacia su entorno social (y por tanto de su responsabilidad social corporativa). Resulta complejo diferenciar ambos escenarios, ya que el enfoque en cada caso depende de las perspectivas utilizadas por los autores y cómo utilizan el concepto de responsabilidad social corporativa.

Se puede decir que la era moderna de la responsabilidad social corporativa comienza en los años cincuenta (Carroll, 1999), tomando después especial protagonismo en los años sesenta (Wang et al., 2016) y siendo objeto de interés por parte de investigadores y empresarios, de manera incremental, desde entonces (Garriga y Melé, 2004). La intensidad de la investigación sobre RSC experimenta actualmente un nuevo repunte con un notable incremento desde el comienzo del Siglo XXI (Montiel y Delgado-Ceballos, 2014). Esto permite valorar positivamente su relevancia actual y proyección a futuro.

\subsection{Enfoques y perspectivas de estudio}

Los motivos por los que una empresa decide incorporar iniciativas de RSC en su actividad habitual pueden ser altruistas o filantrópicos, pero también de tipo estratégico o como herramienta defensiva (Vogel, 2006). El marco regulatorio existente potencia la RSC definiendo unos mínimos en materia de actuación social por parte de las empresas y facilitando referentes en cuanto a las prioridades que se deben abordar. Este marco en muchas ocasiones limita de manera incómoda la actuación de las empresas, tanto en entornos de mercado como de no mercado, y por eso es importante el carácter voluntario de la RSC y los comportamientos de imitación entre empresas (Orlitzky et al., 2003). Las prácticas de RSC también son potenciadas por estándares y certificaciones en materia de responsabilidad social o medioambiental, que conducen a los responsables de empresa a auto-regularse y cumplir mínimamente, a veces incluso de manera simbólica, con sus requisitos (Christmann y Taylor, 2006; Tenbrunsel et al., 2000). Es de esperar que en entornos con presiones normativas o regulatorias débiles las empresas opten de manera general por un «cumplimiento oportunista» mínimo de los requisitos legales (Schwartz y Carroll, 2003, p. 511).

Muchos enfoques o perspectivas de análisis de la RSC se basan en el estudio de los motivos que tienen las empresas o los objetivos que persiguen con la adopción de prácticas de RSC. Principalmente existen dos perspectivas de estudio: instrumental o normativa. Entre ambos extremos se encuentra un amplio espectro continuo basado en las diversas posibles combinaciones de factores (Garriga y Melé, 2004).

La perspectiva con enfoque instrumental, o práctico, considera la RSC como un medio que la empresa utiliza para alcanzar o mejorar sus pro- 
pios resultados. Según este enfoque de tipo económico de la RSC, las actuaciones sociales de la empresa se deben circunscribir exclusivamente a maximizar los beneficios de sus accionistas, interaccionando con otros grupos de interés únicamente cuando estos puedan influir en lo anterior (Friedman, 1970; Goodpaster, 1991). De acuerdo al enfoque instrumental, la RSC no es un tipo de responsabilidad moral, sino una iniciativa de carácter estratégico que puede mejorar los resultados de la empresa (McWilliams et al., 2006) y las relaciones entre negocio y la sociedad, ganando reputación y legitimidad (Dhanesh, 2012). La estrategia de RSC se define como «acciones voluntarias de RSC que mejoran la competitividad y la reputación», dando como resultado de dichas actividades una mejora en el rendimiento económico y financiero (Orlitzky et al., 2011, p. 6).

La perspectiva opuesta presenta un importante componente moral y ético en los fundamentos de la actuación empresarial, utilizando aspectos normativos explícitos en sus planteamientos (Lindgreen y Swaen, 2010). El enfoque normativo hace referencia a «lo que las empresas deberían o no hacer» (Swanson, 1999, p. 506), y a cómo deberían ser. La filosofía normativa está centrada en la evaluación moral y el juicio de la actuación humana. Desde un enfoque normativo, se asume que las empresas no están libres de las obligaciones morales o éticas atribuibles a cualquier miembro de la sociedad (Bauman y Skitka, 2012) y no pueden eludir su responsabilidad de ser una institución social aportando bienes y servicios de tipo económico a la vez que la creación de bienestar para la sociedad. La perspectiva normativa encaja en la visión de la RSC planteada en la Teoría de los Grupos de Interés presentada por Freeman (1984), según la cual, la empresa debe atender las reclamaciones y exigencias de todo el amplio ecosistema de grupos de interés que pueden influir en la empresa, y no solo de aquellos relacionados con los aspectos económicos y financieros de la misma (T. M. Jones, 1995). La empresa debe tener como propósito la coordinación entre los intereses de los grupos de interés (Donaldson y Preston, 1995) persiguiendo, así, el beneficio de la sociedad y los grupos de interés en conjunto.

Mientras que una adopción forzada de prácticas de RSC debida a presiones externas de la sociedad o marcos regulatorios (de tipo reactivo) se considera generalmente de carácter instrumental, cuando las empresas adoptan de modo voluntario la iniciativa RSC (de manera proactiva) lo pueden realizar tanto con objetivos económicos de naturaleza instrumental como con motivos éticos o morales de carácter normativo (Aguinis y Glavas, 2012). En relación a la iniciativa de la empresa, en numerosas ocasiones ocurre que cuando se habla, simplemente, de RSC se suele hacer con un enfoque altruista que no persigue resultados económicos, mientras que cuando se habla de estrategia RSC, normalmente se refiere a un enfoque que busca la rentabilidad (Baron, 2001).

\subsection{Principios de actuación}

Un principio expresa algo fundamental que la gente cree que es verdad, un valor básico que motiva a las personas para actuar (Wood, 1991). La RSC actúa como un conjunto de principios sobre los que las empresas construyen políticas, procesos y generan resultados, es decir, actúan como la base de la construcción del modelo de RSC para cada caso concreto. Desde una perspectiva instrumental, los principios relacionados con aspectos económicos y legales serían fundamentales comportándose como umbrales básicos de funcionamiento de obligado cumplimiento sin los cuales la empresa no es operativa 
(Pinkston y Carroll, 1996). A partir de estos, una actitud ética y proactiva aporta un margen de seguridad ante la llegada de nuevas regulaciones o controversias en la actuación de la empresa, y permite posicionarse de manera favorable ante los deseos y exigencias de la sociedad. Por encima de los niveles económico y legal se colocarían, en primer lugar, la responsabilidad ética, de esperado cumplimiento por parte de la sociedad y sus grupos de interés, y por último, sobre todos los anteriores, la responsabilidad discrecional filantrópica, de deseado cumplimiento, que transforma a la empresa en un buen ciudadano corporativo (Schwartz y Carroll, 2003).

Desde la perspectiva normativa, respecto a la instrumental, se invierte el orden de prioridad en el cumplimiento de los principios de RSC (véase Kang y Wood, 1995), desplazando el componen- te económico desde su posición principal a la última en grado de importancia. Con este enfoque, resultan prioritarias las responsabilidades morales, seguidas de las legales y, por último, las económicas. Según Wood (1991), la llamada responsabilidad discrecional (nivel individual) es prioritaria, a continuación, las empresas deben cumplir con la responsabilidad pública que abarca la faceta legal y la faceta ética (nivel organizacional) y por último la empresa deberá cumplir con las responsabilidades de tipo económicas (nivel institucional). De esta manera, la rentabilidad económica no es posible para una empresa sin cumplir previamente con las obligaciones morales y legales, o estaría adoptando una actitud fraudulenta y perdería su legitimidad para existir. Cada principio tiene asociado un nivel de análisis que facilita su comprensión (ver Figura 1).

\section{Figura 1 - Evolución de principios de la RSC}

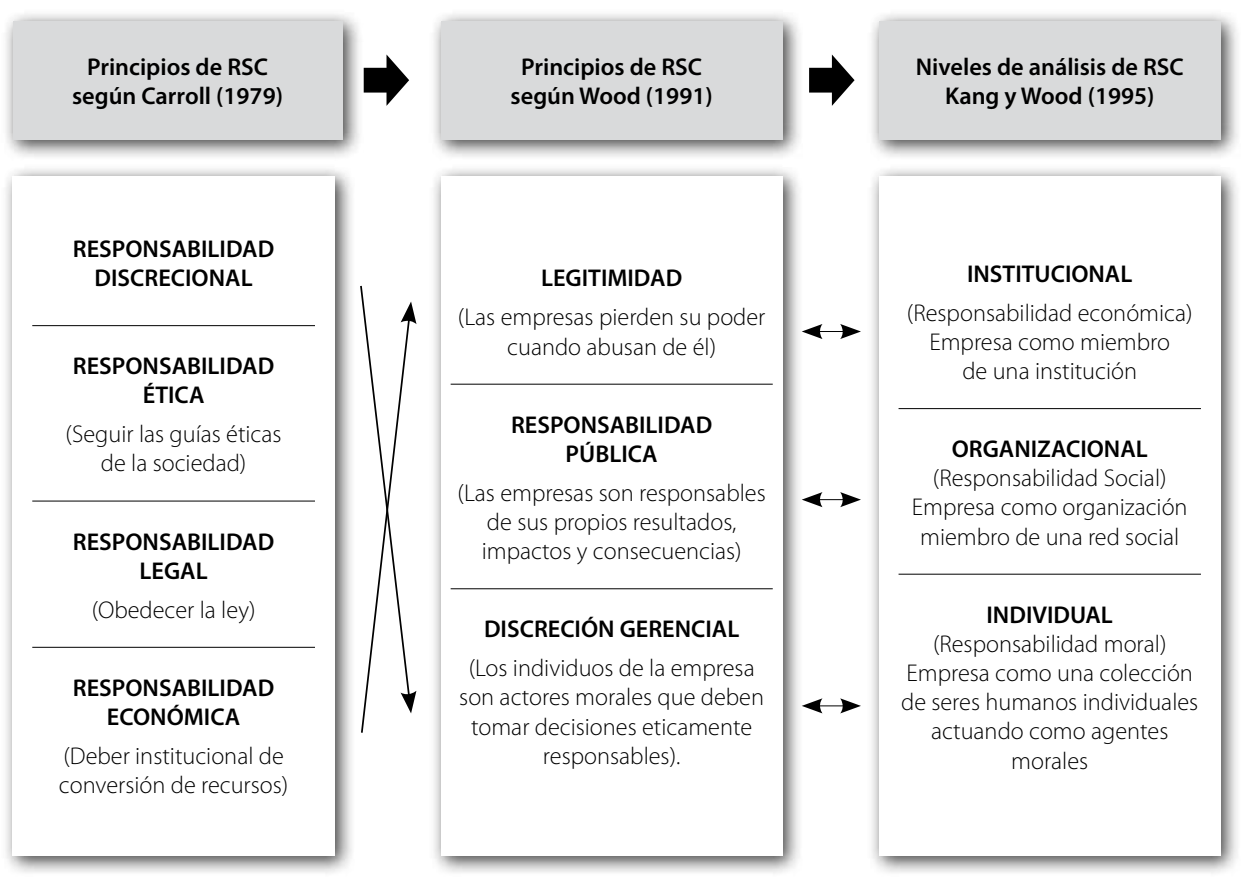




\section{La respuesta social corporativa}

Respuesta social corporativa se define como «los procesos mediante los cuales se producen, supervisan, evalúan, compensan y rectifican (o no) esos resultados [referido a impactos y resultados para la sociedad, grupos de interés y la empresa]» (Wood, 2010, p. 54). Se trata de una manifestación de los planteamientos de RSC de la organización (lo que deben hacer) relacionada con cómo las organizaciones interactúan y gestionan activamente sus entornos (Swanson, 1999). Abarca sus procesos y modos de gestión de incidencias sociales, y su capacidad (lo que pueden hacer), modo de actuación y respuesta ante las demandas sociales (Brammer y Pavelin, 2006; Carroll, 1979; Frederick, 1994). La respuesta social está relacionada con la capacidad para responder a condiciones sociales cambiantes (Wartick y Cochran, 1985). Por capacidad en RSC se entiende el conjunto formado por conocimiento, habilidades y procesos de una empresa relacionado con la planificación, la implementación y la evaluación de la actividad RSC (Lee et al., 2013).

La respuesta social complementa, pero no sustituye, el enfoque normativo y motivacional de la RSC (Wood, 1991) añadiendo una selección de medios, innovaciones y prácticas (contrapunto de acción) para la consecución de los objetivos deseados (Swanson, 1999). Se trata de un concepto de tipo ecológico, que sugiere la adaptación de la empresa a un entorno cambiante (Wood, 1991) y es eminentemente de carácter descriptivo sobre los procesos directivos de la empresa (Carroll, 1979): no analiza la ética de la actuación en cuanto a deber o responsabilidad (Wood, 2010), ya que una empresa podría realizar actuaciones socialmente responsables persiguiendo intereses propios y no generales para la sociedad (Swanson, 1999). Permite a la empresa minimizar la diferencia entre el actual nivel de rendimiento social de una empresa (ver concepto en el apartado siguiente) y el rendimiento que espera la sociedad (Garriga y Melé, 2004), y juega un papel importante en las relaciones con los grupos de interés, especialmente los grupos de interés primarios, permitiendo a los directivos obtener aportaciones constructivas por parte de éstos (Brammer y Pavelin, 2006).

La respuesta social corporativa se orienta principalmente al desarrollo de procesos de toma de decisión organizacionales a través de los cuales, considerando las limitaciones propias de información incompleta e imperfecta, los responsables corporativos de manera colectiva anticipan, responden y gestionan las respuestas a los grupos de interés mediante políticas y prácticas corporativas (Epstein, 1987, p. 104). Los procesos de gestión, de toma de decisiones, y la cantidad de recursos dedicados a ellos, afectan a la capacidad de gestión de incidencias sociales, y derivan en el nivel de respuesta social de la empresa. Con estos enfoques, los principios sociales de la empresa y la conceptualización de RSC pasan a un segundo plano frente a las propias prácticas de RSC y sus procesos (Wood, 2010). Los procesos de respuesta social permiten dirigir la empresa en la dirección que sus propios valores marquen, por ejemplo, hacia la búsqueda del beneficio social o no, caracterizando el comportamiento de la organización para la obtención de resultados sociales (Swanson, 1999).

\section{El rendimiento social corporativo}

Se puede definir el rendimiento social corporativo como «un conjunto de categorizaciones descriptivas de la actividad empresarial, centradas en los impactos y los resultados para la sociedad, los grupos de interés y la empresa» (Wood, 2010, p. 54). Equivale a la captura en un momento 
puntual del agregado de la actuación social de una empresa (Barnett, 2007), y en general hace referencia al volumen de esfuerzo dedicado a la obtención de un desempeño socialmente responsable, y especialmente, a la calidad de dicho desempeño. Otorga a la RSC una estructura, permitiendo, a través de sus prácticas, identificar, promulgar y evaluar responsabilidades de la empresa, o testar deberes concretos afrontados, procesos definidos, resultados obtenidos e impactos generados en individuos, grupos de interés, sociedad y la propia empresa (Wood, 2010). En general facilita resolver la cuestión de la medición de la RSC al considerarse, ésta última, un conjunto de principios o motivación para la actuación social de las empresas (Carroll, 1979) difícilmente medibles.
Wood (1991) reenfoca los principios de la RSC propuestos por Carroll (1979) encajando en un único modelo (ver Figura 2) principios de RSC, procesos de respuesta social (esfuerzo) y resultados del comportamiento de la empresa (Garriga y Melé, 2004), que se corresponden con motivos, comportamientos y resultados de la empresa (Wood, 1991). De acuerdo a este modelo, la responsabilidad social corporativa es el marco y principios de la actuación estratégica en cuestiones sociales (un marco de partida que no evalúa sus resultados). El modelo permite hacer operativa la RSC identificando relaciones causaefecto en situaciones en que, por ejemplo, se combinen buenos resultados basados en malas motivaciones o buenas motivaciones instrumentadas mediante malos procesos.

\section{Figura 2 - Modelo de rendimiento social de Wood (1991)}

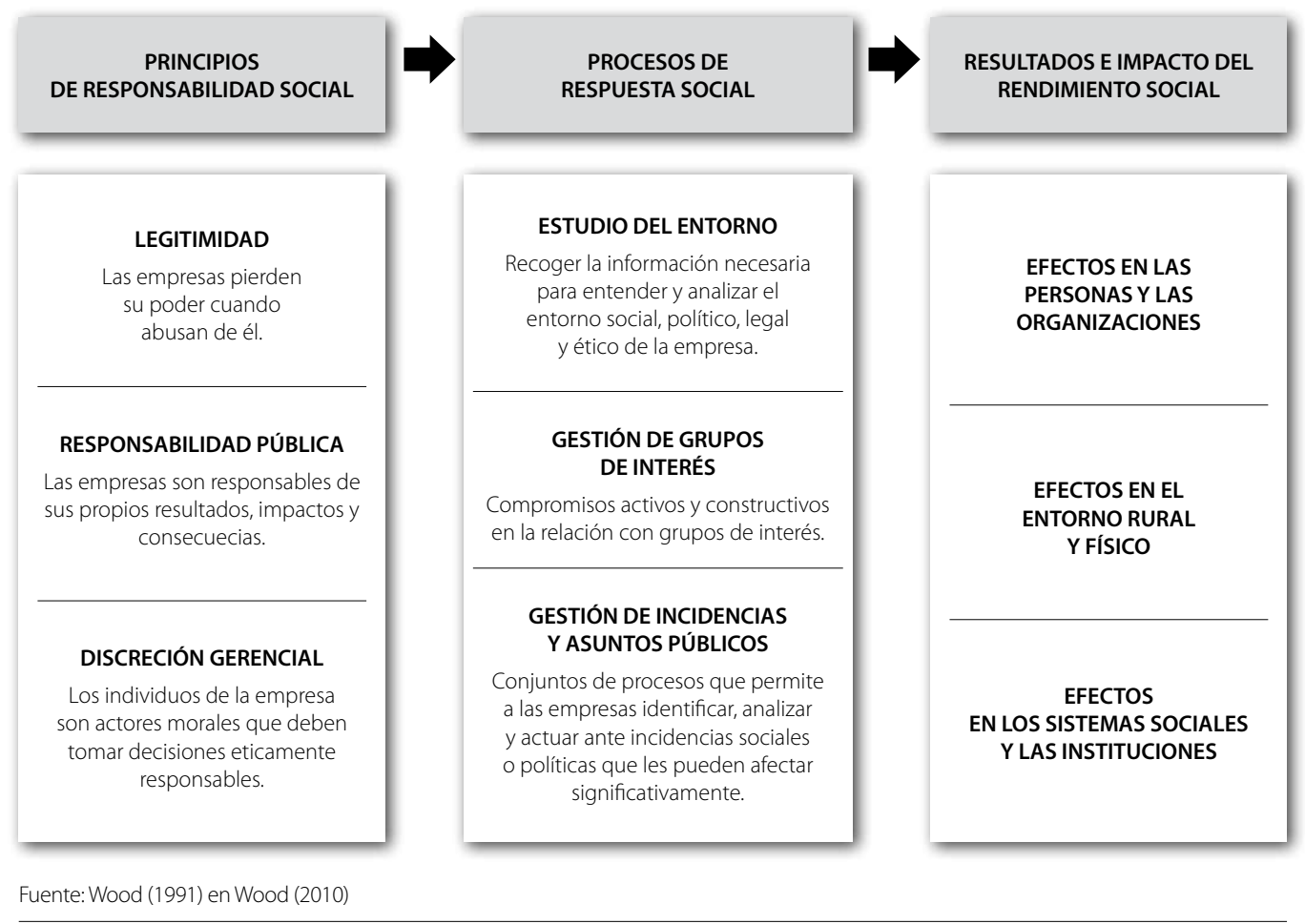




\section{La responsabilidad medioambiental corporativa}

La responsabilidad medioambiental corporativa (RMC) se focaliza en las actividades empresariales, tanto restrictivas como preventivas, que limitan el efecto adverso de la actuación empresarial en el medioambiente ( $\mathrm{N}$. Rahman y Post, 2012). Está relacionada con el deber de la empresa de hacerse cargo de las implicaciones medioambientales de sus operaciones, productos, servicios e instalaciones; eliminar los desechos y la emisión de los mismos; maximizar la eficiencia y productividad de sus recursos; y minimizar prácticas que pudieran afectar negativamente al uso de los recursos por futuras generaciones (Mazurkiewicz, 2004). En general, el concepto de RMC se aplica a empresas que «voluntariamente integran cuestiones medioambientales en sus operaciones de negocio y sus interacciones con los grupos de interés», contribuyendo a un desarrollo sostenible a través de la búsqueda de un equilibrio para minimizar el impacto medioambiental, evitando perjuicios en los rendimientos económicos (N. Rahman y Post, 2012, p. 310, interpretando a Williamson et al., 2006). Debido a que todas las actividades que realiza la empresa afectan de algún modo al medioambiente, se puede decir que éstas están en todo caso incluidas dentro del marco de gestión ambiental que la empresa desee desarrollar (Waddock, 2004). Así, la RMC se puede interpretar como la agenda medioambiental de la empresa y sus resultados, reduciendo impactos negativos en el medio ambiente e incrementando los positivos (Post et al., 2011).

El enfoque predominante de la RMC en el periodo entre 1960 y 1978 consiste en la reducción del impacto medioambiental de las prácticas económicas de las empresas. Posteriormente desde 1980 hasta 1999 el enfoque se centró en el consumo sostenible de los recursos naturales y una producción verde. Actualmente está orientado a su inclusión dentro de las prácticas de responsabilidad social como parte de la relación de la empresa con los grupos de interés (Ganescu y Dindire, 2014).

Se podría decir que la vinculación entre las dimensiones social y medioambiental de la responsabilidad corporativa no es de paralelismo, sino que una de las dos incluye o complementa a la otra. Existen planteamientos según los cuales, la dimensión medioambiental cobra mayor importancia, estando la dimensión social relegada a la primera. Este caso se corresponde con un enfoque de la actividad empresarial llamado ecocentrismo, en el que personas y todos sus sistemas están integrados como partes de la naturale$z a, y$ las actuaciones de las personas deben estudiarse en relación con el entorno natural (Bansal y Roth, 2000, p. 733).

En general, cuando se tiene en consideración el enfoque de la responsabilidad social de la empresa, lo habitual es considerar que la RMC es una parte o dimensión integral y muy importante de la RSC (Aguilera et al., 2007; Bansal y Roth, 2000; Cai et al., 2016; Guenther et al., 2006; Gunningham, 2009; Holtbrugge y Dogl, 2012; Kim et al., 2015; Park y Levy, 2014; Post et al., 2011) y que al hablar de la RSC se habla implícitamente de sus componentes social y medioambiental (Castka et al., 2004) o de «dos tipos» de RSC (Baughn et al., 2007). Esta idea se resume en la definición de RSC ofrecida por la Comisión Europea (European Commission, 2001, p. 6), según la cual la RSC es «un concepto por el cual las empresas integran las preocupaciones sociales y medioambientales en sus operaciones comerciales y en su interacción con sus grupos de interés de manera voluntaria». Otro motivo para la consideración de la RMC como parte de la RSC 
es una interpretación amplia de que la empresa, como agente social, debe asumir una serie de responsabilidades con el medio ambiente, tal y como ocurre con un ciudadano, y que de los resultados de su actitud se beneficiará de manera general la sociedad al completo. El medio natural puede llegar a ser considerado uno más de los grupos de interés sobre los que la empresa debe rendir cuentas (Lynes y Andrachuk, 2008). Por estos motivos, algunos autores se refieren al componente medioambiental de la RSC utilizando el término responsabilidad social corporativa medioambiental (Guenther et al., 2006; Lyon y Maxwell, 2008; Mazurkiewicz, 2004; Post et al., 2011; N. Rahman y Post, 2012), y otros incluso utilizan datos de responsabilidad medioambiental como proxy para el estudio de fenómenos relacionados con la RSC (Orlitzky et al., 2003).

$\mathrm{Al}$ compartir estructura, la RMC se relaciona con conceptos similares a la RSC, como es el caso de la respuesta medioambiental o ecológica corporativa, que se define como «un conjunto de iniciativas corporativas que pretenden mitigar el impacto de una empresa en el medio natural» (Bansal y Roth, 2000, p. 717), y que está relacionada con la naturaleza e intensidad de las prácticas de medioambiente de la empresa. Por otra parte, el rendimiento medioambiental, o rendimiento medioambiental corporativo, es el comportamiento de la empresa hacia el medio natural y los resultados o impactos que la gestión empresarial tienen sobre el mismo (Bhattacharyya y Cummings, 2015).

\section{Otros conceptos relacionados}

En entornos académicos y profesionales se han acuñado un conjunto de términos, distintos a RSC, que representan enfoques de negocio con connotaciones sociales, humanas, éticas o medioambientales. Dahlsrud (2008), en su estudio de las dimensiones presentes en las definiciones de RSC, destaca que un alto porcentaje de ellas incluyen los componentes social, económico y medioambiental. Aquellos conceptos que vinculen el compromiso empresarial con estos tres mismos componentes tendrían una naturaleza próxima al concepto de RSC, mostrando ciertas similitudes. Estos conceptos, cercanos a RSC, pueden actuar en ciertas circunstancias como términos equivalentes o complementarios a RSC. Entre ellos se incluyen (ver Tabla 1 con algunas definiciones representativas): desarrollo sostenible, ciudadanía corporativa, emprendimiento responsable, negocio responsable, triple línea base, sostenibilidad corporativa, responsabilidad corporativa y ética de negocios (Aguilera et al., 2007; Carroll y Shabana, 2010; Dahlsrud, 2008; Farooq et al., 2014; Frederick, 1960; Lin et al., 2012; Park y Levy, 2014; Valor, 2005; Van Marrewijk, 2003; Waddock, 2004).

\section{Tabla 1. Conceptos similares a RSC}

\begin{tabular}{|c|c|c|c|}
\hline Concepto & Autor/es & Definición & Etiquetas \\
\hline $\begin{array}{l}\text { Desarrollo } \\
\text { Sostenible }\end{array}$ & $\begin{array}{l}\text { World Commission } \\
\text { on Environment } \\
\text { and Development } \\
\text { (1987) }\end{array}$ & $\begin{array}{l}\text { Proceso de cambio en el cual la explotación de } \\
\text { recursos, la dirección de las inversiones, la orientación } \\
\text { del desarrollo tecnológico y el cambio institucional } \\
\text { están todos en armonía, y cubre el potencial actual } \\
\text { y futuro de cumplir con las necesidades y aspiraciones } \\
\text { humanas. } \\
\text { Cumplir con las necesidades del presente sin compro- } \\
\text { meter la habilidad de futuras generaciones de cubrir } \\
\text { sus necesidades. }\end{array}$ & $\begin{array}{l}\text { Preservación de recursos. } \\
\text { Largo plazo. } \\
\text { Gestión del cambio. } \\
\text { Planteamiento global. }\end{array}$ \\
\hline
\end{tabular}




\begin{tabular}{|c|c|c|c|}
\hline Concepto & Autor/es & Definición & Etiquetas \\
\hline $\begin{array}{l}\text { Ética de } \\
\text { los Negocios }\end{array}$ & $\begin{array}{l}\text { Epstein } \\
(1987, \text { p. 104) }\end{array}$ & $\begin{array}{l}\text { «La ética de los negocios se refiere a la reflexión } \\
\text { sistemática y basada en el valor de los directivos, } \\
\text { tradicionalmente de manera individual pero cada vez } \\
\text { más en entornos colectivos, sobre el significado moral } \\
\text { de la acción empresarial personal y organizativa y sus } \\
\text { consecuencias para los grupos de interés de la sociedad. } \\
\text { La reflexión moral es fundamental para el concepto de } \\
\text { ética de los negocios». }\end{array}$ & $\begin{array}{l}\text { Discrecionalidad. } \\
\text { Entorno moral. } \\
\text { Valores. }\end{array}$ \\
\hline \multirow{3}{*}{$\begin{array}{l}\text { Ciudadanía Cor- } \\
\text { porativa }\end{array}$} & $\begin{array}{l}\text { European } \\
\text { Commission } \\
(2001, \text { p. 24) }\end{array}$ & $\begin{array}{l}\text { «La gestión de la totalidad de las relaciones entre una } \\
\text { empresa y sus comunidades de acogida, a nivel local, } \\
\text { nacional y mundial». }\end{array}$ & \multirow{3}{*}{$\begin{array}{l}\text { Perfil de actuación. } \\
\text { Agente social. } \\
\text { Preservación de derechos. } \\
\text { Civismo. }\end{array}$} \\
\hline & $\begin{array}{l}\text { Maignan y Ferrell } \\
(2001, \text { p. 458) }\end{array}$ & $\begin{array}{l}\text { «Ciudadanía corporativa designa las actividades adop- } \\
\text { tadas por las empresas para integrar las demandas } \\
\text { sociales en sus actividades». }\end{array}$ & \\
\hline & $\begin{array}{l}\text { Matten y Crane } \\
(2005, \text { p. } 173)\end{array}$ & $\begin{array}{l}\text { «La ciudadanía corporativa describe el papel de } \\
\text { la corporación en la administración de los derechos de } \\
\text { ciudadanía para los individuos». }\end{array}$ & \\
\hline \multirow{2}{*}{$\begin{array}{l}\text { Responsabilidad } \\
\text { Corporativa }\end{array}$} & $\begin{array}{l}\text { Waddock y Bodwell } \\
(2004, \text { p. } 25)\end{array}$ & $\begin{array}{l}\text { «Responsabilidad corporativa se define como las } \\
\text { maneras en las cuales las prácticas operativas de la } \\
\text { empresa (políticas, procesos y procedimientos) afectan } \\
\text { a sus grupos de interés y al medioambiente natural». }\end{array}$ & \multirow{2}{*}{$\begin{array}{l}\text { Transversalidad. } \\
\text { Social y Medioambiental. } \\
\text { Requisitos de grupos de } \\
\text { interés. }\end{array}$} \\
\hline & $\begin{array}{l}\text { Greenwood (2007, } \\
\text { p. } 315 \text { ) }\end{array}$ & $\begin{array}{l}\text { «Responsabilidad Corporativa viene a significar la } \\
\text { responsabilidad de la empresa para actuar de acuerdo } \\
\text { a los intereses de los [llamados] grupos de interés cor- } \\
\text { porativos legítimos». }\end{array}$ & \\
\hline \multirow{2}{*}{$\begin{array}{l}\text { Sostenibilidad } \\
\text { Corporativa }\end{array}$} & Bansal (2005) & $\begin{array}{l}\text { El concepto de desarrollo sostenible corporativo traslada } \\
\text { al nivel de la empresa los tres principios del desarrollo } \\
\text { sostenible, que son integridad medioambiental, pros- } \\
\text { peridad económica y equidad social. }\end{array}$ & \multirow{2}{*}{$\begin{array}{l}\text { Equilibrio económico, so- } \\
\text { cial y medioambiental. } \\
\text { Largo plazo. } \\
\text { Crecimiento. }\end{array}$} \\
\hline & $\begin{array}{l}\text { Neubaum y Zahra } \\
(2006, \text { p. } 111)\end{array}$ & $\begin{array}{l}\text { «Capacidad de una empresa para nutrir y apoyar } \\
\text { el crecimiento a través del tiempo mediante el } \\
\text { cumplimiento eficaz de las expectativas de los diversos } \\
\text { grupos de interés». }\end{array}$ & \\
\hline $\begin{array}{l}\text { Sostenibilidad } \\
\text { Ecológica }\end{array}$ & $\begin{array}{l}\text { Starik y Rands } \\
\text { (1995) }\end{array}$ & $\begin{array}{l}\text { «La sostenibilidad ecológica es la capacidad de una o } \\
\text { más entidades, ya sea individual o colectivamente, de } \\
\text { existir y florecer (sin cambios o en formas evoluciona- } \\
\text { das) para largos periodos de tiempo, de tal manera que } \\
\text { la existencia y el florecimiento de otras colectividades } \\
\text { de entidades está permitido en niveles y sistemas } \\
\text { relacionados». }\end{array}$ & $\begin{array}{l}\text { Co-existencia. } \\
\text { Biodiversidad. } \\
\text { Preservación del entorno } \\
\text { natural y social. }\end{array}$ \\
\hline \multirow{2}{*}{ Triple Línea Base } & $\begin{array}{l}\text { Elkington } \\
(1997)\end{array}$ & $\begin{array}{l}\text { Escenario en el que las empresas armonizan sus } \\
\text { esfuerzos y recursos para la obtención de resultados } \\
\text { económicamente viables, mientras son a la vez social y } \\
\text { medioambientalmente responsables. }\end{array}$ & \multirow{2}{*}{$\begin{array}{l}\text { Rendición de cuentas. } \\
\text { Equilibrio económico, so- } \\
\text { cial y medioambiental. } \\
\text { Largo plazo. }\end{array}$} \\
\hline & $\begin{array}{l}\text { European } \\
\text { Commission } \\
(2001, \text { p. 26) }\end{array}$ & $\begin{array}{l}\text { «ldea de que el rendimiento general de una empresa } \\
\text { debe medirse en función de su contribución combi- } \\
\text { nada a la prosperidad económica, la calidad ambiental } \\
\text { y el capital social». }\end{array}$ & \\
\hline
\end{tabular}


Dependiendo del enfoque utilizado, investigadores y profesionales con iniciativas empresariales que incluyen dimensiones sociales y medioambientales pudieran preferir referirse a RSC o alguno de los conceptos expuestos en la Tabla 1. A continuación se desarrollan aquellos con mayor presencia en literatura académica y prácticas empresariales.

\subsection{Responsabilidad corporativa}

La responsabilidad corporativa destaca la necesidad de buscar un equilibrio entre los elementos que la componen: económico, social y medioambiental (Montiel y Delgado-Ceballos, 2014). La responsabilidad corporativa, definida como «políticas y acciones de las organizaciones, para contextos específicos, que tienen en cuenta las expectativas de los grupos de interés y la triple línea base de rendimiento económico, social y medioambiental» (Aguinis, 2011, p. 855), viene a significar que todas las prácticas de la empresa tienen efectos sobre grupos de interés y el medio ambiente, y que la empresa debe gestionar las responsabilidades generadas debido a ello, integrando a los grupos de interés y el medioambiente en su estrategia (Waddock, 2004). Este concepto resulta similar a RSC cuando en la definición de ésta última se considera el aspecto medioambiental como una responsabilidad más de la empresa hacia la sociedad, y se puede utilizar este término para sustituir al de RSC cuando ocurre esta circunstancia.

En cuanto a similitudes con otros conceptos, según el planteamiento de van Marrewijk (2003), la responsabilidad corporativa abarca los aspectos sociales, medioambientales y económicos de manera similar a la Triple Linea Base, por lo que pueden considerarse conceptos equivalentes. Para otros autores, responsabilidad corporativa de una empresa es un concepto similar al de ciu- dadanía corporativa (Donaldson, 1996; Marsden, 2000).

\subsection{Ciudadanía corporativa}

El término ciudadanía corporativa ha sido adoptado principalmente por agentes del sector profesional (directivos y empresarios). Mientras que la RSC incorpora aspectos morales, gerenciales y sociológicos, la ciudadanía corporativa se centra en un aspecto más estrecho relacionado con las actividades que la empresa adopta para cumplir con las demandas de la sociedad de manera responsable (Maignan et al., 1999). A la empresa se le asigna el papel de un buen o mal ciudadano, adquiriendo el concepto un enfoque de carácter político (Matten y Crane, 2005).

En términos de ciudadanía corporativa, una empresa es reactiva cuando rechaza las responsabilidades asignadas por sus grupos de interés intentando evitar tener que cumplirlas, moviéndose a un entorno menos exigente o influyendo en ellas mediante, por ejemplo, prácticas de lobby. Con esta condición una empresa no sería un buen ciudadano corporativo (Maignan et al., 1999). En cambio, una empresa es proactiva, y por lo tanto es un ciudadano corporativo ejemplar, cuando las cumple sistemáticamente e incluso se anticipa a estas demandas (Maignan et al., 1999; Maignan y Ferrell, 2001). Este concepto, con su dimensión de civismo, resalta el hecho de que la empresa tiene un papel como agente social cuya actuación afecta a otros miembros de la sociedad y a sus derechos de ciudadano (Matten y Crane, 2005). La empresa se considera un garante o promotor de derechos para las personas, en aquellos aspectos en que administración, reguladores o gobiernos, no tienen alcance (Crane y Matten, 2016). Por ejemplo, una empresa global cuyo ámbito geográfico de actuación sobrepasa las regulaciones estatales, debería aplicar sus cri- 
terios éticos de manera absoluta en todas las áreas o regiones de afectación, como si de un ciudadano global se tratase (Rupp et al., 2011).

Respecto del enfoque de la RSC, la ciudadanía corporativa tiene una visión más práctica de la actividad empresarial y su relación con el entorno. Resalta el hecho de que las empresas son actores públicos poderosos que tienen derechos y obligaciones y que deben respetar los derechos de otros ciudadanos (especialmente los reales). Conecta la actividad empresarial con el plano social para el mutuo beneficio (Valor, 2005).

\subsection{Sostenibilidad corporativa}

Los conceptos de sostenibilidad corporativa y desarrollo sostenible aparecen estrechamente vinculados (Sharma y Henriques, 2005) compartiendo la perspectiva de crecimiento, el componente temporal (especialmente el largo plazo) y un enfoque marcadamente eco-centrista (Gladwin et al., 1995) incorporando medioambiente como valor intrínseco en el modelo que actúa como dimensión complementaria (no accesoria) al resto de dimensiones (Montiel, 2008). Eco-eficiencia se define como «concepto según el cual mejorar la forma en que se utilizan los recursos puede reducir los daños ambientales y reducir costos» (European Commission, 2001, p. 24). En aquellas ideas relacionadas con la sostenibilidad, el medioambiente deja de ser algo externo al modelo para ser uno de los aspectos centrales, o directamente el elemento central, a considerar (Cai et al., 2016). Sostenibilidad corporativa, a su vez, puede tener dos enfoques, uno que combina los factores económico, medioambiental y social, y otro que enfatiza especialmente el aspecto medioambiental, referido al entorno en general, en cuyo caso se aproximaría más al concepto de sostenibilidad ecológica (Montiel, 2008). El enfoque de sostenibilidad corporativa, al igual que otros enfoques de RSC derivados de desarrollo sostenible, expanden el ámbito de actuación medioambiental para incluir la dimensión social como inseparable para la consecución los objetivos en el largo plazo (Watts y Holme, 2000). En estos casos, cuando se habla de sostenibilidad corporativa, la responsabilidad social corporativa se refiere específicamente al ámbito de las personas y las organizaciones (Van Marrewijk, 2003).

Los investigadores en responsabilidad o rendimiento social corporativo analizan el resultado social (incluyendo medioambiente) y el económico de manera separada, mientras que en el caso de la sostenibilidad corporativa se asume una interrelación entre sus tres componentes (económico, medioambiental y social). Se puede decir también que, en aquellos estudios sobre RSC en los que se analiza un equilibrio entre sus dimensiones social y medioambiental, el enfoque utilizado se aproxima al de sostenibilidad corporativa (Montiel, 2008), al igual que ocurre con el concepto responsabilidad corporativa.

Respecto a la relación entre sostenibilidad corporativa y responsabilidad corporativa, algunos autores proponen que el primero podría ocupar un nivel superior, definiendo el grado de inclusión de los asuntos medioambientales y sociales en las operaciones de la empresa y sus relaciones con los grupos de interés (Figura 3). Este nivel de encaje de la responsabilidad corporativa en la empresa y su entorno repercute en la capacidad de las empresas para perdurar y generar beneficios en el largo plazo (Van Marrewijk, 2003), ya que permite a la empresa adaptarse estratégicamente a los cambios del entorno y considerar la complejidad de su contexto social. Por eso se percibe una relación entre sostenibilidad corporativa y el concepto de sostenibilidad 


\section{Figura $3 \cdot$ Modelo de sostenibilidad corporativa}

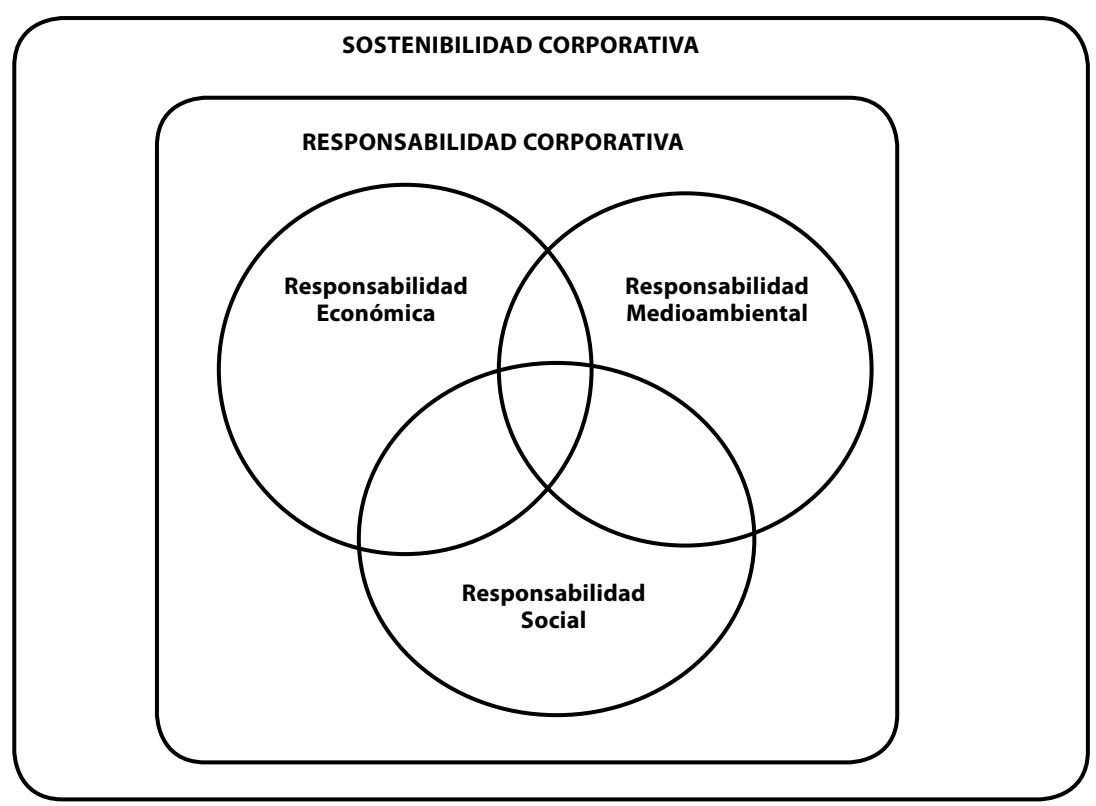

Fuente: van Marrewijk (2003)

(como capacidad de perdurar) de la empresa (Marsden, 2000).

\subsection{Sostenibilidad ecológica}

Sostenibilidad ecológica, también llamada sostenibilidad medioambiental (Van Marrewijk, 2003), consiste en aprovechar los recursos naturales a la vez que se mejora las condiciones de las personas (Starik y Rands, 1995). Mientras se puede decir que sostenibilidad corporativa abarca dimensiones sociales, económicas y medioambientales, también se puede aceptar que sostenibilidad ecológica se refiera específicamente o principalmente a aspectos de medioambiente (Montiel y Delgado-Ceballos, 2014), por lo que no se trataría de conceptos similares (Van Marrewijk, 2003).

\section{Conclusiones}

El concepto de RSC, debido a su propia naturaleza, ha sufrido una evolución en sus enfoques y perspectivas de análisis (Garriga y Melé, 2004; S. Rahman, 2011). Aunque actualmente presenta un cierto grado de maduración, todavía muestra ambigüedad y falta de acuerdo en ambientes profesionales y académicos (Aguinis y Glavas, 2012; Sheehy, 2015). Se complementa con otra serie de conceptos relacionados más concretos y con mayor aplicación práctica que se deben conocer. Una correcta comprensión de estos conceptos y sus relaciones es básica para la adecuada aplicación de los planteamientos, estrategias y políticas en materia de actuación social y medioambiental.

Se podría decir que el nivel de exigencia por parte de los agentes sociales para que las empre- 
sas operen en marcos de actividad socialmente responsables es cada vez mayor (Yim y Fock, 2013). Las empresas, como agentes del sistema socio-económico, deben ganarse, para poder operar, el respeto de los grupos de interés, considerando que éstas son instadas a perseguir estrategias socialmente responsables (Brammer et al., 2007). Esta situación empuja a las empresas a retratarse en cuanto a su posicionamiento en aspectos sociales, haciéndolo visible mediante sus prácticas (estrategias, políticas y programas). Tanto las empresas, a nivel organizativo, como sus directivos, a nivel discrecional, deben mantener un equilibrio entre las críticas que demandan más responsabilidad social y los inversores que puedan exigir mayores beneficios en el corto plazo, empujándoles a buscar estrategias rentables que incorporen un importante componente filantrópico o nuevos planteamientos con componentes más éticos (Porter y Kramer, 2006).

La publicidad, promociones y otro tipo de comunicaciones pueden ayudar a difundir la imagen de empresa como un agente social preocupado por las necesidades sociales de los grupos de interés (Maignan y Ferrell, 2004). Sin embargo, el alineamiento entre la imagen social corporativa y la identidad corporativa es clave para conseguir una reducción del escepticismo que pueda generarse entre los consumidores, para lo cual es necesario entender y analizar detenidamente la estrategia de RSC de la compañía y sus componentes (Pomering y Johnson, 2009).

Es importante mantener la coherencia entre el posicionamiento social de la empresa y la comunicación que ésta realiza sobre el mismo. Cuando el encaje o similitud entre el mensaje publicitario y la imagen de marca es mayor, y la empresa es bien conocida, el consumidor reacciona más favorablemente a los mensajes y las marcas (Nan y
Heo, 2007). Igualmente ocurre cuando la misión de la empresa y el tipo de iniciativa social que se publicita coinciden, generando un comportamiento negativo por parte del consumidor en caso contrario (Becker-Olsen et al., 2006). Cuanto más expone la empresa su actividad y posicionamiento social, más atrae la atención crítica de los grupos de interés, hasta el punto de que pueden llegar a sospechar que con mucha actividad publicitaria, la empresa podría intentar esconder algo (Morsing y Schultz, 2006). Este efecto se ve potenciado por la existencia de grupos de interés, como los consumidores, que cuando están familiarizados y sensibilizados con la RSC reaccionan de manera especialmente positiva a la comunicación de la misma por parte de la empresa (Klein y Dawar, 2004). En muchas ocasiones resulta inevitable rendir cuentas a los consumidores, ya que grupos activistas o asociaciones pueden participar en reducir la información asimétrica, alertando al consumidor sobre la verdadera actuación y motivaciones de las empresas (Feddersen y Gilligan, 2001). También los propios empleados, ejerciendo como grupo de interés primarios de la compañía especialmente sensible a la comunicación social de la misma (Drumwright, 1996), pueden contribuir a desmantelar la credibilidad de dicha comunicación. Las empresas, por tanto, no pueden eludir la tarea de conocer, evaluar, analizar, proponer e incorporar un planteamiento estratégico social y unos principios éticos, basándose en los enfoques y perspectivas presentados en ese artículo, considerando que puedan éstos resultar familiares a sus consumidores u otros grupos de interés.

Las empresas deben identificar sus grupos de interés, entre los que se encuentran sus públicos objetivos (internos o externos), cada cual con sus propias expectativas. En base a ellos las empresas deben estudiar su reputación y, en caso 
necesario, definir o modificar sus principios de RSC. Estos principios pueden plantearse desde una perspectiva instrumental, en cuyo caso sería necesario evaluar su estrategia, o desde una perspectiva normativa, en cuyo caso se necesitaría evaluar, además, los valores en la empresa (Garriga y Melé, 2004). En cualquiera de las dos opciones, es interesante recordar que los grupos de interés, en general, valoran el compromiso social de la empresa en base a su actuación visible (relacionada con su respuesta social corporativa) y el impacto que generan (vinculado con el concepto de rendimiento social corporativo). Por ello, independiente de lo que la empresa comunique, son más importantes los actos y la efectividad de la empresa (Wood, 2010). La percepción de algún tipo de incongruencia entre los principios empresariales y sus actos puede tener un impacto especialmente perjudicial para la reputación de la empresa.

En un planteamiento contemporáneo de la responsabilidad empresarial, el componente económico es uno más de los tres pilares de creación de valor (Montiel, 2008) que la empresa debe desarrollar (y no el único). Este planteamiento queda retratado de acuerdo a los postulados de la llamada triple línea base que sirven de referencia para valorar la importancia de este triple equilibrio en la creación de valor por parte de la empresa (Elkington, 1997).

Desde la década de los noventa existe una especial sensibilidad por parte del público general hacia aspectos relacionados con el medioambiente y el impacto de la actividad empresarial y la globalización como causante de su deterioro. El modelo industrial clásico asociado a las empresas ha venido históricamente acompañado de dicho efecto. La concienciación sobre ciertos problemas globales como el cambio climático, el agotamiento de los recursos naturales o el dete- rioro de entornos naturales ha provocado que el componente medioambiental de la CSR haya cobrado protagonismo en los negocios (Auld et al., 2008; Park y Levy, 2014). La problemática surgida respecto a los asuntos de sostenibilidad medioambiental, así como la actuación de grupos de interés como gobiernos, consumidores o comunidades locales han forzado a las empresas a reconocer la importancia de lo medioambiental y a modificar su agenda de actuación para convertirse en organizaciones medioambientalmente responsables (Setthasakko, 2009). Este es uno de los motivos por los que surge una nueva corriente de conceptos como la sostenibilidad corporativa y la sostenibilidad ecológica, amparados por los planteamientos del desarrollo sostenible, en los que el medioambiente ocupa una posición central (enfoque eco-céntrico), y se fomentan los planteamientos de crecimiento en el largo plazo, con especial atención igualmente a aspectos de derechos humanos e impacto en comunidades. Las empresas que adoptan este enfoque para minimizar o eliminar su impacto medioambiental, afectan positivamente, de manera colateral a las personas (véase, por ejemplo, González-Masip, 2016).

Los planteamientos corporativos basados en RSC, para ser efectivos y creíbles, deben aspirar a tener un alcance transversal en la compañía (valores corporativos), y deben incluir el componente medioambiental como dimensión de importancia (Montiel y Delgado-Ceballos, 2014). En circunstancias así, el uso del concepto responsabilidad corporativa es una manera cómoda de referirse a responsabilidad social corporativa, aunque sería necesario valorar si éste último goza de una mayor difusión y aceptación entre los grupos de interés objetivo y, por lo tanto, puede resultar conveniente utilizarlo en las comunicaciones corporativas. 
Otros conceptos, como el de ciudadanía corporativa, se han desarrollado reforzando el planteamiento de la empresa como agente social que actúa en un contexto de derechos de las personas y se comporta con nivel deseable de ética y civismo (Maignan et al., 1999; Moon et al., 2005). Este concepto tiene un enfoque de carácter político y antropo-céntrico, y es más común en entornos profesionales, asignando a la empresa la cualidad de buen ciudadano en el ecosistema social. Pretende analizar el comportamiento ético y las actuaciones empresariales, tiene en consideración el impacto que éstos puedan tener en la percepción y evaluación de sus grupos de interés, y está más orientado al cumplimiento de las demandas exigidas por el entorno de manera reactiva.

El concepto de responsabilidad social corporativa (o sus equivalentes) no debe plantearse únicamente para cuestiones de imagen o reputación empresarial, ya que su credibilidad se contrasta de manera relativamente sencilla mediante la evaluación de la actuación y el rendimiento social de la empresa. Actualmente goza de relevancia estratégica, especialmente en aquellos escenarios en que la conciencia medioambiental está firmemente establecida o en ámbitos sociales notablemente críticos o sensibles a prácticas poco éticas o escándalos. Debido a la globalidad y visibilidad de los impactos y el empeoramiento del medio ambiente, existe una especial sensibilización por parte de los grupos de interés en este aspecto, por ello el esfuerzo de las empresas por minimizar su impacto en el entorno natural debe quedar patente. La utilización de otros conceptos que colocan el componente medioambiental como núcleo de mayor importancia puede resultar de interés, aunque no se debe olvidar el poner en valor el impacto positivo que este tipo de actuaciones sigue teniendo sobre personas y comunidades.

Futuras líneas de investigación podrían orientarse hacia la identificación de nuevos términos o conceptos, en la literatura académica o profesional, que impliquen vinculación o actuación de la empresa con aspectos sociales o medioambientales, y señalar, en los mismos, parecidos o semejanzas con los conceptos revisados en este artículo. Sería interesante también detectar la mayor presencia o predilección de uno u otro enfoque, y cuáles de ellos generan mayores efectos o vinculación con el consumidor a través de actuaciones de comunicación de la empresa que visibilicen su compromiso, actividad e impacto social. 


\section{Bibliografía}

Ackerman, R. W. (1973). How companies respond to social demands. Harvard business review, 51(4), 88-98.

Aguilera, R. V., Rupp, D. E., Williams, C. A. y Ganapathi, J. (2007). Putting the S Back in Corporate Social Responsibility: A Multilevel Theory of Social Change in Organizations. Academy of Management. The Academy of Management Review, 32(3), 836-863.

Aguinis, H. (2011). Organizational responsibility: Doing good and doing well. En S. Zedeck (Ed.), APA handbook of industrial and organizational psychology (pp. 855-879). Washington: American Psychological Association.

Aguinis, H. y Glavas, A. (2012). What We Know and Don't Know About Corporate Social Responsibility: A Review and Research Agenda. Journal of Management, 38(4), 932-968.

Albinger, H. S. y Freeman, S. J. (2000). Corporate Social Performance and Attractiveness as an Employer to Different Job Seeking Populations. Journal of Business Ethics, 28(3), 243.

Auld, G., Bernstein, S. y Cashore, B. (2008). The new corporate social responsibility. Annual Review of Environment and Resources, 33, 413-435.

Bagnoli, M. y Watts, S. G. (2003). Selling to socially responsible consumers: Competition and the private provision of public goods. Journal of Economics \& Management Strategy, 12(3), 419-445.

Bansal, P. y Roth, K. (2000). Why companies go green: A model of ecological responsiveness. Academy of Management Journal, 43(4), 717-736.

Bansal, P. (2005). Evolving Sustainably: A Longitudinal Study of Corporate Sustainable Development. Strategic Management Journal, 26(3), 197-218.

Barnett, M. L. (2007). Stakeholder influence capacity and the variability of financial returns to corporate social responsibility. The Academy of Management Review, 32(3), 794-816.

Baron, D. P. (2001). Private politics, corporate social responsibility, and integrated strategy. Journal of Economics $E$ Management Strategy, 10(1), 7-45.

Baron, D. P. (2008). Managerial contracting and corporate social responsibility. Journal of Public Economics, 92(1), 268-288.

Baughn, C. C., Bodie, N. L. y McIntosh, J. C. (2007). Corporate social and environmental responsibility in Asian countries and other geographical regions. Corporate Social Responsibility and Environmental Management, 14(4), 189.
Bauman, C. W. y Skitka, L. J. (2012). Corporate social responsibility as a source of employee satisfaction. Research in Organizational Behavior, 32, 63-86.

Becker-Olsen, K. L., Cudmore, B. A. y Hill, R. P. (2006). The impact of perceived corporate social responsibility on consumer behavior. Journal of Business Research, 59(1), 46-53.

Behrend, T. S., Baker, B. A. y Thompson, L. F. (2009) Effects of Pro-Environmental Recruiting Messages: The Role of Organizational Reputation. Journal of Business and Psychology, 24(3), 341-350.

Bhattacharyya, A. y Cummings, L. (2015). Measuring Corporate Environmental Performance - Stakeholder Engagement Evaluation. Business Strategy and the Environment, 24(5), 309.

Boulding, K. E. (1956). General systems theory-the skeleton of science. Management science, 2(3), 197-208.

Bowen, H. R. (1953). Social responsibilities of the businessman. New York: Harper.

Brammer, S. J., Millington, A. y Rayton, B. (2007). The contribution of corporate social responsibility to organizational commitment. The International Journal of Human Resource Management, 18(10), 1701.

Brammer, S. J. y Pavelin, S. (2006). Corporate Reputation and Social Performance: The Importance of Fit. The Journal of Management Studies, 43(3), 435-455.

Cai, L., Cui, J. y Jo, H. (2016). Corporate Environmental Responsibility and Firm Risk. Journal of Business Ethics, 139(3), 563-594.

Carroll, A. B. (1979). A Three-Dimensional Conceptual Model of Corporate Performance. Academy of Management. The Academy of Management Review, 4(4), 497.

Carroll, A. B. (1999). Corporate social responsibility. Business and Society, 38(3), 268-295.

Carroll, A. B. y Shabana, K. M. (2010). The Business Case for Corporate Social Responsibility: A Review of Concepts, Research and Practice. International Journal of Management Reviews, 12(1), 85-105.

Castka, P., Balzarova, M. A., Bamber, C. J. y Sharp, J. M. (2004). How can SMEs effectively implement the CSR agenda? A UK case study perspective. Corporate Social Responsibility and Environmental Management, 11(3), 140-149. 
Christmann, P. y Taylor, G. (2006). Firm self-regulation through international certifiable standards: determinants of symbolic versus substantive implementation. Journal of International Business Studies, 37(6), 863-878.

Cochran, P. L. (2007). The evolution of corporate social responsibility. Business horizons, 50(6), 449-454.

Crane, A. y Matten, D. (2016). Business ethics: Managing corporate citizenship and sustainability in the age of globalization Oxford University Press.

Dahlsrud, A. (2008). How corporate social responsibility is defined: an analysis of 37 definitions. Corporate Social Responsibility and Environmental Management, 15(1), 1-13.

Davis, K. (1960). Can Business Afford to Ignore Social Responsibilities? California Management Review (pre-1986), 2(3), 70.

Dhanesh, G. S. (2012). The view from within: internal publics and CSR. Journal of Communication Management, 16(1), 39-58.

Dodd, E. M. (1932). For Whom Are Corporate Managers Trustees? Harvard law review, 45(7), 1145-1163.

Donaldson, T. (1996). Values in tension: Ethics away from home. Harvard business review, 74(5), 48-\&.

Donaldson, T. y Preston, L. E. (1995). The Stakeholder Theory of the Corporation: Concepts, Evidence, and Implications. Academy of Management. The Academy of Management Review, 20(1), 65.

Drumwright, M. E. (1996). Company advertising with a social dimension: The role of noneconomic criteria. The Journal of Marketing, 60(4), 71-87.

Du, S., Bhattacharya, C. y Sen, S. (2010). Maximizing Business Returns to Corporate Social Responsibility (CSR): The Role of CSR Communication. International Journal of Management Reviews, 12(1), 8-19.

Elkington, J. (1997). Cannibals with forks. Oxford, UK: Capstone.

Epstein, E. M. (1987). The corporate social policy process: Beyond business ethics, corporate social responsibility, and corporate social responsiveness. California management review, 29(3), 99-114.

European Commission (2001). Green Paper. Promoting a European framework for Corporate Social Responsibility. Brussels: European Commission.
Farooq, O., Payaud, M., Merunka, D. y Valette-Florence, P. (2014). The Impact of Corporate Social Responsibility on Organizational Commitment: Exploring Multiple Mediation Mechanisms. Journal of Business Ethics, 125(4), 563580.

Feddersen, T. J. y Gilligan, T. W. (2001). Saints and markets: Activists and the supply of credence goods. Journal of Economics \& Management Strategy, 10(1), 149-171.

Fombrun, C. y Shanley, M. (1990). What's in a Name? Reputation Building and Corporate Strategy. The Academy of Management Journal, 33(2), 233-258.

Fox, A. (2007). Corporate Social Responsibility Pays Off. HRMagazine, 52(8), 43-47.

Frederick, W. C. (1994). From CSR1 to CSR2 the maturing of business-and-society thought. Business \& Society, 33(2), 150-164.

Frederick, W. C. (1960). The Growing Concern over Business Responsibility. California Management Review (pre1986), 2(000004), 54.

Freeman, R. E. (1984). Strategic Management: A Stakeholder Approach. Boston, MA: Pittman.

Friedman, M. (1970). The social responsibility of business is to increase its profits. The New York Times Magazine,

Gallardo-Vázquez, D. y Sánchez-Hernández, M. I. (2014). Structural analysis of the strategic orientation to environmental protection in SMEs. BRQ Business Research Quarterly, 17(2), 115-128.

Ganescu, C. y Dindire, L. (2014). Corporate environmental responsibility - a key determinant of corporate reputation. Computational Methods in Social Sciences, 2(1), 48-53.

Garriga, E. y Melé, D. (2004). Corporate Social Responsibility Theories: Mapping the Territory. Journal of Business Ethics, 53(1-2), 51-71.

Gladwin, T. N., Kennelly, J. J. y Krause, T. (1995). Shifting paradigms for sustainable development: Implications for management theory and research. Academy of Management Review, 20(4), 874-907.

González-Masip, J. (2016). Gestión hotelera y sostenibilidad medioambiental: el caso de NH Hoteles. Economía industrial, (401), 141-152.

González-Masip, J. (2017). Efectos de las prácticas de responsabilidad social y medioambiental corporativas en la atracción y 
retención de talento. (No publicada Universidad Complutense de Madrid, Madrid.

Goodpaster, K. E. (1991). Business ethics and stakeholder analysis. Business ethics quarterly, 1(01), 53-73.

Greening, D. W. y Turban, D. B. (2000). Corporate social performance as a competitive advantage in attracting a quality workforce. Business and Society, 39(3), 254-280.

Greenwood, M. (2007). Stakeholder Engagement: Beyond the Myth of Corporate Responsibility. Journal of Business Ethics, 74(4), 315-327.

Guenther, E., Hoppe, H. y Poser, C. (2006). Environmental corporate social responsibility of firms in the mining and oil and gas industries: Current status quo of reporting following GRI guidelines. Greener Management International, (53), $7-25$

Gunningham, N. (2009). Shaping corporate environmental performance: a review. Environmental Policy and Governance, 19(4), 215-231.

Holtbrugge, D. y Dogl, C. (2012). How international is corporate environmental responsibility? A literature review. Journal of International Management, 18(2), 180-195.

Hsu, K. (2012). The advertising effects of corporate social responsibility on corporate reputation and brand equity: Evidence from the life insurance industry in Taiwan. Journal of Business Ethics, 109(2), 189-201.

Hussain, S. S. (1999). The ethics of 'going green': the corporate social responsibility debate. Business Strategy and the Environment, 8(4), 203-210.

Jones, D. A., Willness, C. R. y Madey, S. (2014). Why are job seekers attracted by corporate social performance? Experimental and field tests of three signal-based mechanisms. Academy of Management Journal, 57(2), 383-404.

Jones, T. M. (1995). Instrumental stakeholder theory: A synthesis of ethics and. Academy of Management. The Academy of Management Review, 20(2), 404.

Kang, Y., \& Wood, D. J. (1995). Before-profit social responsibility: Turning the economic paradigm upside down. Proceedings of the International Association for Business and Society, , 6 809-829.

Kilcullen, M. y Kooistra, J. O. (1999). At least do no harm: sources on the changing role of business ethics and corporate social responsibility. Reference Services Review, 27(2), 158-178.
Kim, H., Park, K. y Ryu, D. (2015). Corporate environmental responsibility: A legal origins perspective. Journal of Business Ethics,

Klein, J. y Dawar, N. (2004). Corporate social responsibility and consumers' attributions and brand evaluations in a product-harm crisis. International Journal of research in Marketing, 21(3), 203-217.

Knudsen, J. S. (2013). The integration of corporate social responsibility (CSR) initiatives into business activities: can lessons be learnt from gender diversity programmes? International Journal of Business Governance and Ethics, 8(3), 210

Lee, E. M., Park, S. y Lee, H. J. (2013). Employee perception of CSR activities: Its antecedents and consequences. Journal of Business Research, 66(10), 1716.

Levitt, T. (1958). The dangers of social-responsibility. Harvard business review, 36(5), 41-50.

Lichtenstein, D. R., Drumwright, M. E. y Braig, B. M. (2004). The effect of corporate social responsibility on customer donations to corporate-supported nonprofits. Journal of Marketing, 68(4), 16-32.

Lin, C., Baruch, Y. y Shih, W. (2012). Corporate Social Responsibility and Team Performance: The Mediating Role of Team Efficacy and Team Self-Esteem. Journal of Business Ethics, 108(2), 167-180.

Lindgreen, A. y Swaen, V. (2010). Corporate Social Responsibility. International Journal of Management Reviews, 12(1) $1-7$.

Lynes, J. K. y Andrachuk, M. (2008). Motivations for corporate social and environmental responsibility: a case study of Scandinavian Airlines. Journal of international management, 14(4), 377-390

Lyon, T. P. y Maxwell, J. W. (2008). Corporate social responsibility and the environment: A theoretical perspective. Review of environmental economics and policy, 2(2), 240-260.

Maignan, I. y Ferrell, O. C. (2004). Corporate Social Responsibility and Marketing: An Integrative Framework. Journal of the Academy of Marketing Science, 32(1), 3-19.

Maignan, I. y Ferrell, O. C. (2001). Corporate citizenship as a marketing instrument - Concepts, evidence and research directions. European Journal of Marketing, 35(3/4), 457-484.

Maignan, I., Ferrell, O. C. y Hult, G. T. M. (1999). Corporate citizenship: Cultural antecedents and business benefits Journal of the Academy of Marketing Science, 27(4), 455-469. 
Marsden, C. (2000). The new corporate citizenship of big business: Part of the solution to sustainability? Business and Society Review, 105(1), 8-25.

Matten, D. y Moon, J. (2008). Implicit' and 'explicit' CSR: a conceptual framework for a comparative understanding of corporate social responsibility. Academy of Management Review, 33(2), 404-24.

Matten, D. y Crane, A. (2005). Corporate citizenship: Toward an extended theoretical conceptualization. Academy of Management. The Academy of Management Review, 30(1), 166-179.

Mazurkiewicz, P. (2004). Corporate environmental responsibility: Is a common CSR framework possible. Consultado en http://documents.worldbank.org/curated/en/5770514 68339093024/pdf/421830csfframework01PUBLICl.pdf

McGuire, J. W. (1969). THE CHANGING NATURE OF BUSINESS RESPONSIBILITIES. Journal of Risk and Insurance (pre-1986), 36(1), 55.

McWilliams, A. y Siegel, D. (2001). Corporate social responsibility: A theory of the firm perspective. Academy of Management. The Academy of Management Review, 26(1), 117-127.

McWilliams, A., Siegel, D. S. y Wright, P. M. (2006). Corporate social responsibility: strategic implications. Journal of Management Studies, 43(1), 1-18.

Montiel, I. (2008). Corporate Social Responsibility and Corporate Sustainability: Separate Pasts, Common Futures. Organization \& Environment, 21(3), 245-269.

Montiel, I. y Delgado-Ceballos, J. (2014). Defining and Measuring Corporate Sustainability: Are We There Yet? Organization \& Environment, 27(2), 113-139.

Moon, J., Crane, A. y Matten, D. (2005). CAN CORPORATIONS BE CITIZENS: Corporate Citizenship as a Metaphor for Business Participation in Society. Business Ethics Quarterly, 15(3), 429-453.

Morsing, M. y Schultz, M. (2006). Corporate social responsibility communication: stakeholder information, response and involvement strategies. Business Ethics: A European Review, 15(4), 323-338.

Nan, X. y Heo, K. (2007). Consumer responses to corporate social responsibility (CSR) initiatives: Examining the role of brand-cause fit in cause-related marketing. Journal of advertising, 36(2), 63-74.
Neubaum, D. O. y Zahra, S. A. (2006). Institutional ownership and corporate social performance: The moderating effects of investment horizon, activism, and coordination. Journal of Management, 32(1), 108-131.

Nicholls, S. y Kang, S. (2012). Going green: the adoption of environmental initiatives in Michigan's lodging sector. Journal of Sustainable Tourism, 20(7), 953.

Orlitzky, M., Schmidt, F. L. y Rynes, S. L. (2003). Corporate social and financial performance: A meta-analysis. Organization Studies, 24(3), 403-441.

Orlitzky, M., Siegel, D. S. y Waldman, D. A. (2011). Strategic Corporate Social Responsibility and Environmental Sustainability. Business and Society, 50(1), 6.

Park, S. y Levy, S. E. (2014). Corporate social responsibility: perspectives of hotel frontline employees. International Journal of Contemporary Hospitality Management, 26(3), 332-348.

Pinkston, T. S. y Carroll, A. B. (1996). A Retrospective Examination of CSR Orientations: Have They Changed? Journal of Business Ethics, 15(2), 199.

Pomering, A. y Johnson, L. W. (2009). Advertising corporate social responsibility initiatives to communicate corporate image: Inhibiting scepticism to enhance persuasion. Corporate Communications: An International Journal, 14(4), 420-439.

Porter, M. E. y Kramer, M. R. (2006). Strategy \& Society: The Link between Competitive Advantage and Corporate Social Responsibility. Harvard Business Review, 84(12), 78-92.

Post, C., Rahman, N. y Rubow, E. (2011). Green Governance: Boards of Directors' Composition and Environmental Corporate Social Responsibility. Business and Society, 50(1), 189.

Price, L. L., Feick, L. F. y Guskey, A. (1995). Everyday market helping behavior. Journal of Public Policy \& Marketing, 14(2), 255.

Rahman, N. y Post, C. (2012). Measurement Issues in Environmental Corporate Social Responsibility (ECSR): Toward a Transparent, Reliable, and Construct Valid Instrument. Journal of Business Ethics, 105(3), 307-319.

Rahman, S. (2011). Evaluation of definitions: ten dimensions of corporate social responsibility. World Review of Business Research, 1(1), 166-176.

Rupp, D. E., Williams, C. A., \& Aguilera, R. V. (2011). Increasing corporate social responsibility through stakeholder value internalization (and the catalyzing effect of new governance): An application of organizational justice, self-de- 
termination, and social influence theories. En M. Schminke (Ed.), Managerial ethics: Managing the psychology of morality () Routledge/Psychology Press.

Schwartz, M. S. y Carroll, A. B. (2003). Corporate Social Responsibility: A Three-Domain Approach. Business Ethics Quarterly, 13(4), 503-530.

Sen, S. y Bhattacharya, C. B. (2001). Does doing good always lead to doing better? Consumer reactions to corporate social responsibility. Journal of Marketing Research, $38(2), 225-243$

Setthasakko, W. (2009). Barriers to implementing corporate environmental responsibility in Thailand: A qualitative approach. International Journal of Organizational Analysis, 17(3), 169-183.

Sharma, S. y Henriques, I. (2005). Stakeholder influences on sustainability practices in the Canadian forest products industry. Strategic Management Journal, 26(2), 159-180.

Sheehy, B. (2015). Defining CSR: Problems and solutions. Journal of Business Ethics, 131(3), 625-648.

Siegel, D. S. y Vitaliano, D. F. (2007). An empirical analysis of the strategic use of corporate social responsibility. Journal of Economics \& Management Strategy, 16(3), 773-792.

Starik, M. y Rands, G. P. (1995). Weaving an integrated web: Multilevel and multisystem perspectives of ecologically sustainable organizations. Academy of Management Review, 20(4), 908-935.

Swanson, D. L. (1999). Toward an integrative theory of business and society: A research strategy for corporate social performance. Academy of Management. The Academy of Management Review, 24(3), 506-521.

Tenbrunsel, A. E., Wade-Benzoni, K. A., Messick, D. M. y Bazerman, M. H. (2000). Understanding the influence of environmental standards on judgments and choices. Academy of Management Journal, 43(5), 854-866.

Valor, C. (2005). Corporate Social Responsibility and Corporate Citizenship: Towards Corporate Accountability. Business and Society Review, 110(2), 191.

Van Marrewijk, M. (2003). Concepts and definitions of CSR and corporate sustainability: Between agency and communion. Journal of Business Ethics, 44(2/3), 95-105.
Vogel, D. (2006). The Market for Virtue: The Potential and Limits of Corporate Social Responsibility (2a ed.). Washington, DC: Brookings Institution Press.

Waddock, S. (2004). Parallel Universes: Companies, Academics, and the Progress of Corporate Citizenship. Business and Society Review: Journal of the Center for Business Ethics at Bentley College, 109(1), 5-42.

Waddock, S. y Bodwell, C. (2004). Managing Responsibility: What can be learned from the quality movement? California management review, 47(1), 25-37.

Waddock, S., Bodwell, C. y Graves, S. B. (2002). Responsibility: The new business imperative. The Academy of Management Executive, 16(2), 132-148

Waddock, S. y Graves, S. B. (1997). The corporate social performance-financial performance link. Strategic Management Journal, 18(4), 303-319.

Wagner, T., Lutz, R. J. y Weitz, B. A. (2009). Corporate hypocrisy: Overcoming the threat of inconsistent corporate social responsibility perceptions. Journal of Marketing, 73(6), 77-91.

Wang, H., Tong, L., Takeuchi, R. y George, G. (2016). Corporate Social Responsibility: An Overview and New Research Directions. Academy of Management Journal, 59(2) 534.

Wartick, S. L. y Cochran, P. L. (1985). The Evolution of the Corporate Social Performance Model. The Academy of Management Review, 10(4), 758.

Watts, P. y Holme, L. (2000). Corporate Social Responsibility: Making Good Business Sense. Geneva: World Business Council for Sustainable Development.

Weber, M. (2008). The business case for corporate social responsibility: A company-level measurement approach for CSR. European Management Journal, 26(4), 247-261.

Whetten, D. A., Rands, G., \& Godfrey, P. (2002). What are the responsibilities of business to society? En A. Pettigrew, H. Thomas \& R. Whittington (Eds.), Handbook of strategy and management (pp. 373-408). London: Sage.

Williamson, D., Lynch-Wood, G. y Ramsay, J. (2006). Drivers of Environmental Behaviour in Manufacturing SMEs 